\title{
High virulence does not necessarily impede viral adaptation to a new host: a case study using a plant RNA virus
}

\author{
Anouk Willemsen ${ }^{1,2^{*}}$ D, Mark P. Zwart ${ }^{1,3}$ and Santiago F. Elena ${ }^{1,4}$
}

\begin{abstract}
Background: Theory suggests that high virulence could hinder between-host transmission of microparasites, and that virulence therefore will evolve to lower levels. Alternatively, highly virulent microparasites could also curtail host development, thereby limiting both the host resources available to them and their own within-host effective population size. In this case, high virulence might restrain the mutation supply rate and increase the strength with which genetic drift acts on microparasite populations. Thereby, this alternative explanation limits the microparasites' potential to adapt to the host and ultimately the ability to evolve lower virulence. As a first exploration of this hypothesis, we evolved Tobacco etch virus carrying an eGFP fluorescent marker in two semi-permissive host species, Nicotiana benthamiana and Datura stramonium, for which it has a large difference in virulence. We compared the results to those previously obtained in the natural host, Nicotiana tabacum, where we have shown that carriage of eGFP has a high fitness cost and its loss serves as a real-time indicator of adaptation.

Results: After over half a year of evolution, we sequenced the genomes of the evolved lineages and measured their fitness. During the evolution experiment, marker loss leading to viable virus variants was only observed in one lineage of the host for which the virus has low virulence, D. stramonium. This result was consistent with the observation that there was a fitness cost of eGFP in this host, while surprisingly no fitness cost was observed in the host for which the virus has high virulence, $N$. benthamiana. Furthermore, in both hosts we observed increases in viral fitness in few lineages, and host-specific convergent evolution at the genomic level was only found in N. benthamiana.

Conclusions: The results of this study do not lend support to the hypothesis that high virulence impedes microparasites' evolution. Rather, they exemplify that jumps between host species can be game changers for evolutionary dynamics. When considering the evolution of genome architecture, host species jumps might play a very important role, by allowing evolutionary intermediates to be competitive.
\end{abstract}

Keywords: Adaptation, Experimental evolution, Genome architecture evolution, Host-pathogen interactions, Virulence, Virus evolution

\section{Background}

From both applied and fundamental perspectives, virulence is a key phenotypic trait of microparasites. In medicine and agriculture, it is crucial to understand mechanistically how microparasites harm the host, in

\footnotetext{
* Correspondence: anouk.willemsen@ird.fr

${ }^{1}$ Instituto de Biología Molecular y Celular de Plantas (IBMCP), Consejo Superior de Investigaciones Científicas-Universidad Politécnica de Valencia, Campus UPV CPI 8E, Ingeniero Fausto Elio s/n, 46022 València, Spain 2Present address: MIVEGEC (UMR CNRS 5290, IRD 224, UM), National Center for Scientific Research (CNRS), 911 Avenue Agropolis, BP 64501, 34394 Cedex 5 Montpellier, France

Full list of author information is available at the end of the article
}

order to devise effective interventions. From a more fundamental perspective, evolutionary biologists have long been interested in understanding why many microparasites are highly virulent. It has been suggested that virulence reduces between-host transmission, and that selection would therefore act to maximize between-host transmission by reducing virulence $[1,2]$. High virulence would signal maladaptation, for example following a host-species jump, and eventually be selected against. The ubiquity of microparasitic virulence and the fact that many apparently well-adapted microparasites have high virulence led to a more sophisticated framework: 
the hypothesis that there are tradeoffs between virulence and transmission [2-5]. This framework posits that high levels of replication could increase the probability of a microparasite being transferred to a new host, whilst also increasing the probability that the host would die quickly and the temporal window for transmission would be very brief. Under this more plausible framework, virulence evolves to the level that optimizes between-host transmission $[4,6,7]$.

The tradeoff hypothesis forms the cornerstone for theoretical frameworks considering the evolution of virulence in many different pathosystems. Many important additions to the framework have been made, for example recognizing that within-host competition and opportunism can lead to increases in virulence [8-10]. Moreover, the importance of other factors at the between-host level have been given consideration, such as self-shading [11]. The effects of evolution on microparasitic virulence have therefore been given considerable attention, although the number of experimental studies that address this issue is still rather limited, especially for viruses [12].

Conversely, virulence itself could also have profound effects on evolution, including its own evolutionary dynamics [13]. This reversed causality is already apparent from the tradeoff model, under which microparasites with suboptimal virulence will undergo reduced between-hosts transmission. All else being equal, if a smaller number of hosts are infected, effective population size will be decreased, increasing the strength of genetic drift and decreasing the mutation supply rate. In addition, the evolution to optimum virulence may be slow as this optimum is not static and can shift towards lower virulence as the density of susceptible hosts decreases [14]. Moreover, a wide range of virulence can be associated with each step of evolution towards the optimum, where selection favors genotypes with higher fitness that may improve transmission but not necessarily improve virulence [13]. Besides these effects of virulence on evolution, it is conceivable that a similar within-host effect could also occur, when virulence curtails host development (i.e., host growth or evolution) and thereby limits the host resources available to the microparasite. Virulence would then limit the microparasite effective population size within hosts, again reducing the mutation supply and thereby slowing the rate of adaptation. Interestingly, $(i)$ a low effective population size, $(i i)$ a non-static optimum virulence, (iii) large variations in virulence on the fitness landscape, and (iv) reduced host-development, could limit the rate at which lower virulence evolves, meaning that high virulence might persist longer than suggested by the simple tradeoff model [13].

There are many reasons why high virulence in hostpathogen interactions could emerge, but the most likely avenue is probably a change of host species. For example, infection of Ebola virus in bats is asymptomatic, while in humans and other primates the death rate is high [15]. Changes in virulence have been explained by the host phylogeny, where similar levels of virulence are displayed by closely related hosts and host jumps across large genetic distances may result in high virulence [16]. However, if a microparasite is confronted with a new host environment in which its level of virulence is altered, how does virulence affect its ability to adapt to the new host?

Here we address this question using Tobacco etch virus (TEV; genus Potyvirus, family Potyviridae), a (+)ssRNA virus with a $9.5 \mathrm{~kb}$ genome that infects a wide-range of host plants, and an experimental evolution approach. To consider the effect of virulence (e.g., virus-induced host mortality or reduction in biomass) on virus adaptation, we looked for two natural host species in which $(i)$ there was some evidence that TEV potential for adaptation would be roughly similar, and (ii) there was a large difference in virulence. The distribution of mutational fitness effects (DMFE) of TEV has been compared in eight host species, and this study concluded that there were strong virus genotype-by-host species interactions [17]. For many host species distantly related to the natural host of TEV, Nicotiana tabacum, the DMFE changed drastically; many mutations that were neutral or deleterious in $N$. tabacum, became beneficial. However, for two related host species, Nicotiana benthamiana and Datura stramonium, most mutations tested remained neutral or deleterious [17], implying that the fraction of beneficial mutations in both hosts is small. Moreover, virus accumulation after one week of infection is also similar for both hosts [18]. On the other hand, TEV infection of $N$. benthamiana will typically result in heavy stunting and the death of the plant within a matter of weeks, whereas TEV infection of $D$. stramonium is virtually asymptomatic. These symptoms are very different from symptoms observed for TEV in its natural host $N$. tabacum, which does not lead to plant death but consist of vein clearing, mosaic mottling, chlorosis, and stunting $[19,20]$. Whilst there are many similarities between TEV infections in these two hosts, one key difference is host-pathogen interactions and therewith levels of viral virulence brought about.

As a first exploration of the effects of virulence on microparasite evolution, we therefore decided to evolve TEV in N. benthamiana and D. stramonium. By serially passaging each independent lineage in a single plant every round, our study maximizes within-host selection. This setup allows us to exclusively focus on the effects of within-host selection, although for our model system we expect to see large differences in the resulting population size and the extent of virus movement within the host. Moreover, to immediately gauge whether adaptive 
evolution might be occurring, we passaged a TEV variant expressing a marker protein (Fig. 1), the enhanced GFP (eGFP). This exogenous sequence of 762 nts increases TEV genome to a size just over $10 \mathrm{~kb}$. Upon longduration passages in $N$. tabacum, the eGFP gene is quickly lost due to its strong fitness cost, and its loss is reliably indicated by a loss of eGFP fluorescence [21]. Therefore, we can use the time to eGFP loss in the virus population as a real-time indicator of adaptation. To determine the frequency of eGFP loss and host-specific mutations in each viral population (i.e., lineage), the ancestral virus and evolved lineages were sequenced by Illumina technology. According to the above hypothesis that high virulence may impair the rate of microparasite evolution, we expect that adaptive evolution would occur more quickly in the host species for which TEV has lower virulence, $D$. stramonium, than in the host species for which it has high virulence, $N$. benthamiana. Hence, we expected that in $D$. stramonium (i) the eGFP marker would be lost more rapidly, (ii) there would be more sequence-level convergent evolution, and (iii) there would be larger increases in within-host competitive fitness. However, our experiments did not confirm these simple hypotheses. First of all, the loss of eGFP could not be used as an indicator of adaptation in neither D. stramonium nor N. benthamiana. Second, hostspecific convergent evolution at the genomic level was only found in N. benthamiana. Third, in both hosts we observe only one evolved lineage with increases in within-host competitive fitness, and surprisingly the eGFP marker does not seem to have a fitness cost in $N$. benthamiana. These results were unexpected based on our previous work in N. tabacum [21], but they do exemplify the extent to which a host species jump can be a game changer for RNA virus evolutionary dynamics.

\section{Methods}

Virus stocks, plants and serial passages

The TEV genome used to generate TEV-eGFP virus was originally isolated from $N$. tabacum plants [22]. To generate a virus stock of the ancestral TEV-eGFP, the pMTEV-eGFP plasmid [23] was linearized by digestion with $B g l \mathrm{II}$ prior to in vitro RNA synthesis using the mMESSAGE mMACHINE ${ }^{\circ}$ SP6 Transciption Kit (Ambion), as described in [24]. The third true leaf of 4-week-old $N$. tabacum L var Xanthi $N N$ plants was mechanically inoculated with $5 \mu \mathrm{g}$ of transcribed RNA. All symptomatic tissue was collected 7 days post-inoculation (dpi).

For the serial passage experiments, $500 \mathrm{mg}$ homogenized stock tissue was ground into fine powder and di-

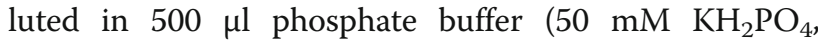
$\mathrm{pH} 7.0,3 \%$ polyethylene glycol 6000 ). From this mixture, $20 \mu \mathrm{l}$ were then mechanically inoculated on the sixth true leaf of 4-week old $N$. benthamiana Domin plants and on the third true leaf of 4-week old D. stramonium $\mathrm{L}$ plants. Ten independent replicates were used for each host plant. Based on a previous study done in N. tabacum [21], passages of TEV-eGFP in D. stramonium were done every 9 weeks. In $N$. benthamiana the virus induces host mortality, and therefore the passages had to be restricted to 6 weeks for this host. At the end of the designated passage duration all leaves above the inoculated one were collected and stored at $-80{ }^{\circ} \mathrm{C}$. For subsequent passages the frozen tissue was homogenized and a sample was ground and resuspended with an equal amount of phosphate buffer [21]. Then, new plants were mechanically inoculated as described above. Three 9-week passages were performed for lineages evolved in D. stramonium (27 weeks of evolution) and five 6-week passages for lineages evolving in $N$. benthamiana (30 weeks of evolution), making the total time of evolution similar in both hosts. For both $D$. stramonium and $N$. benthamiana, all individual plants used were grown from large stocks of seeds collected from selfpollinated individual plants, therefore all plants for each host were of identical genotype. All plants were kept in a biosafety level 2 greenhouse at $24^{\circ} \mathrm{C}$ with $16 \mathrm{~h}$ light:8 $\mathrm{h}$ dark photoperiod.

Reverse transcription polymerase chain reaction (RT-PCR) To determine whether deletions occurred at the $e G F P$ locus, RNA was extracted from $100 \mathrm{mg}$ homogenized infected tissue using the InviTrap Spin Plant RNA Mini Kit (Stratec Molecular). Reverse transcription (RT) was performed using MMuLV reverse transcriptase (Thermo Scientific) and reverse primer 5'-CGCACTACATAGGAGAATTAG-3' located in the 3'UTR of the TEV-eGFP genome (GenBank accession; KC918545, positions 10235-10255). PCR was then performed with Taq DNA polymerase (Roche) and primers flanking the eGFP gene:

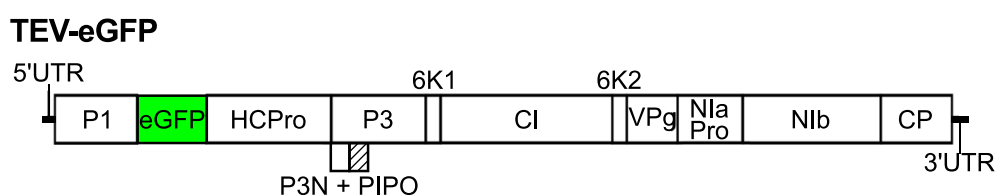

Fig. 1 Schematic representation of TEV-eGFP. The eGFP gene is located between P1 and HC-Pro genes. Proteolytic cleavage sites were provided at both ends of eGFP 
forward 5'-GCAATCAAGCATTCTACTTC-3' (positions 48-67), and reverse 5'-CCTGATATGTTTCCTGATAAC3' (positions 2530-2550). PCR products were resolved by electrophoresis on $1 \%$ agarose gels.

\section{Illumina sequencing, variants, and SNP calling}

For Illumina next-generation sequencing (NGS) of the evolved and ancestral lineages, the viral genomes were amplified by RT-PCR using AccuScript Hi-Fi (Agilent Technologies) reverse transcriptase and Phusion DNA polymerase (Thermo Scientific), with six independent replicates that were pooled. Each virus was amplified using three primer sets, generating three amplicons of similar size (set 1: 5'-GCAATCAAGCATTCTACTTCTA TTGCAGC-3' and 5'-CCTGATATGTTTCCTGATAAC3' (positions 48-76 and 2530-2550); set 2: 5'-ACACGTA CTGGCTGTCAGCG-3' and 5'-CATCAATGTCAATGG TTACAC-3' (positions 2217-2236 and 5324-5344); set 3: 5'-CCCGTGAAACTCAAGATAG-3' and 5'-CGCACTA CATAGGAGAATTAG-3' (positions 5044-5062 and 10235-10255). Equimolar mixtures of the three PCR products were made. Sequencing was performed at GenoScreen (Lille, France: www.genoscreen.com). Illumina HiSeq2500 $2 \times 100$ bp paired-end libraries with dual-index adaptors were prepared along with an internal PhiX control. Libraries were prepared using the Nextera XT DNA Library Preparation Kit (Illumina Inc.). Sequencing quality control was performed by GenoScreen, based on PhiX error rate and Q30 values.

Read artifact filtering and quality trimming (3' minimum Q28 and minimum read length of $50 \mathrm{bp}$ ) was done using FASTX-Toolkit v.0.0.14 [25]. De-replication of the reads and 5' quality trimming requiring a minimum of Q28 was done using PRINSEQ-lite v.0.20.4 [26]. Reads containing undefined nucleotides $(\mathrm{N})$ were discarded. Initially, the ancestral TEV-eGFP sequence was mapped using Bowtie v.2.2.6 [27] against the reference TEV-eGFP sequence (GenBank accession: KC918545). Error correction was done using Polisher v2.0.8 (available for academic use from the Joint Genome Institute) and a consensus sequences was defined for the ancestral TEV-eGFP lineage. Subsequently, the cleaned reads of the evolved sequences were mapped using Bowtie v.2.2.6 against the new defined consensus sequence. Single nucleotide mutations for each viral lineage were identified using SAMtools' mpileup [28] and VarScan v.2.3.9 [29], where the maximum coverage was set to 40000 and mutations with a frequency $<1 \%$ were discarded. Note that the single nucleotide mutations detected here can be fixed (frequency $>50 \%$ ) in the evolved lineages, as the detection was done over the ancestral population. Hence, it allows us to compare the mutations that arose by evolving TEV-eGFP in the different hosts.

\section{Virus accumulation and within-host competitive fitness assays}

Prior to performing assays, the genome equivalents per $100 \mathrm{mg}$ of tissue of the ancestral virus stocks and all evolved lineages were determined for subsequent fitness assays. The InviTrap Spin Plant RNA Mini Kit (Stratec Molecular) was used to isolate total RNA of $100 \mathrm{mg}$ homogenized infected tissue. Real-time quantitative RTPCR (RT-qPCR) was performed using the One Step SYBR PrimeScript RT-PCR Kit II (Takara), in accordance with manufacturer instructions, in a StepOnePlus RealTime PCR System (Applied Biosystems). Specific primers for the coat protein gene $(C P)$ were used: forward 5'TTGGTCTTGATGGCAACGTG-3' (positions 9968-9987) and reverse 5'-TGTGCCGTTCAGTGTCTTCCT-3' (positions 9998-10018). The StepOne Software v.2.2.2 (Applied Biosystems) was used to analyze the data. The concentration of genome equivalents per $100 \mathrm{mg}$ of tissue was then normalized to that of the sample with the lowest concentration, using phosphate buffer.

For the accumulation assays, 4-week-old $N$. benthamiana and D. stramonium plants were mechanically inoculated with $50 \mu \mathrm{l}$ of the normalized dilutions of ground tissue. Inoculation of each viral lineage was done on the same host plant on which it had been evolved, plus TEV and the ancestral TEV-eGFP virus on each of the hosts, using three independent plant replicates per lineage. Leaf tissue was harvested 10 dpi. Total RNA was extracted from $100 \mathrm{mg}$ of homogenized tissue. Virus accumulation was then determined by means of RT-qPCR for the $C P$ gene of the ancestral and the evolved lineages. For each of the harvested plants, at least three technical replicates were used for RT-qPCR.

To measure within-host competitive fitness, we used TEV carrying a red fluorescent protein: TEV-mCherry as a common competitor. This virus has a similar insert size and within-host fitness compared with TEV-eGFP [21]. All ancestral and evolved viral lineages were again normalized to the sample with the lowest concentration, and 1:1 mixtures of viral genome equivalents were made with TEV-mCherry [23]. The mixture was mechanically inoculated on the same host plant on which it had been evolved, plus TEV and the ancestral TEV-eGFP virus on each of the hosts, using three independent plant replicates per viral lineage. The plant leaves were collected at $10 \mathrm{dpi}$, and stored at $-80{ }^{\circ} \mathrm{C}$. Total RNA was extracted from $100 \mathrm{mg}$ homogenized tissue. RT-qPCR for the $C P$ gene was used to determine total viral accumulation, and independent RT-qPCR reactions were also performed for the mCherry sequence (GenBank accession: AY678264) using specific primers: forward 5'-CGGCGAGTTCATC TACAAGG-3' (positions 360 to 379) and reverse 5'-TGG TCTTCTTCTGCATTACGG-3' (positions 416-436). The ratio of the evolved and ancestral lineages to TEV- 
mCherry $(R)$ is then $R=\left(n_{C P^{-}} n_{m C h e r r y}\right) /\left(n_{m C h e r r y}\right)$, where $n_{C P}$ and $n_{m \text { Cherry }}$ are the RT-qPCR measured copy numbers of $C P$ and $m$ Cherry, respectively. Then we can estimate the within-host competitive fitness as $W=\sqrt[t]{R_{t} / R_{o}}$, where $R_{0}$ is the ratio at the start of the experiment and $R_{t}$ the ratio after $t$ days of competition [24]. The statistical analyses comparing the fitness between lineages were performed using R v.3.2.2 [30] and IBM SPSS Statistics version 23.

\section{Results}

Experimental setup and fluorescent marker stability upon passaging of TEV-eGFP

TEV-eGFP was mechanically passaged in $N$. benthamiana and D. stramonium, as described in Zwart et al. 2014 [21]. In this previous study, we noted that 9-week long passages led to rapid deletion of $e G F P$ as well as rapid convergent evolution in N. tabacum [21]. We therefore chose to use similar long passages to maximize the effects of selection in general, and in particular because we intended to use the loss of $e G F P$ as a real time measure of adaptation. Although 9-week passages could be performed in $D$. stramonium, for $N$. benthamiana this was not possible due to virus-induced host mortality. These plants died after 6 weeks of infection, and therefore we were forced to collect tissue at this time point. As $D$. stramonium grows to similar heights as $N$. tabacum when infected with TEV, and $N$. benthamiana does not grow much after infection, we chose to maximize infection duration to make the results comparable to those obtained in N. tabacum [21]. We performed three 9-week passages in D. stramonium and - to keep the total evolutionary time comparable - five 6-week passages in $N$. benthamiana. In $D$. stramonium all ten lineages initiated were completed, whereas in $N$. benthamiana only 6/10 lineages were completed. The remaining four $N$. benthamiana lineages failed to cause infection in subsequent rounds of passaging, and were therefore halted. Initial symptomatology of TEV-eGFP in $N$. benthamiana was very mild, while this symptomatology was more severe in the second and subsequent passages, possibly indicating adaptation of the virus to this alternative host. In $D$. stramonium the symptomatology was constant along the evolution experiment.

Based only on previous results in N. tabacum, we expected that the exogenous $e G F P$ gene sequence would be rapidly purged [21,31,32], and as such would serve as a first indicator of the occurrence of TEV adaptation. However, the usefulness of fluorescence for determining the integrity of the eGFP marker was limited in both hosts, by $(i)$ the high levels of autofluorescence in the highly symptomatic $N$. benthamiana leaves, and (ii) the patchy fluorescence in the D. stramonium tissue. Therefore, unlike for TEV-eGFP in N. tabacum, the fluorescent marker was of limited use here. Nevertheless, all $N$. benthamiana lineages appeared to have some fluorescence until the end of the evolution experiment, and we observed a loss of fluorescence in only $1 / 10 \mathrm{D}$. stramonium lineages in the third 9-week passage.

After each passage, RNA was extracted from the collected leaf tissue, and RT-PCR with primers flanking the eGFP insert was performed. This RT-PCR assay can therefore detect deletions in the eGFP gene, even when deletions extend well into the downstream HC-Pro gene [21]. In general, the RT-PCR results confirmed the fluorescence microscopy results: a large deletion was detected only in the one $D$. stramonium lineage with a loss of fluorescence (Fig. 2a; 9-weeks passage 2 L8). This deletion variant went to a high frequency in the subsequent passage (Fig. 2a; 9-weeks passage 3 L8). For N. benthamiana lineages, we did detect a low-frequency deletion in the $e G F P$ gene in one lineage (Fig. 2b; 6-weeks passage 4 and $5 \mathrm{~L} 4$ ), but this deletion is so large that this variant is most likely no longer capable of autonomous replication. The deletion size is around $1500 \mathrm{nt}$, which means that besides deleting the entire $e G F P$, around 800 nt are deleted from HC-Pro, which has a size of $1377 \mathrm{nt}$ in total. This deletion extends well into the central region of HC-Pro, beyond the well-conserved FRNK box, which is essential for virus movement and RNA-silencing suppressor activity [33, 34]. As complementation by full-length virus variants can slow down the rate at which deleterious variants are eliminated from the virus population [35], we performed an extra round of passaging with all $N$. benthamiana lineages to check whether this variant would remain at a low frequency, and found exactly this result (Fig. 2b; 6-week passage 6 L4). Furthermore, we detected a small deletion in one lineage (Fig. 2b; 6-week passage 5 and $6 \mathrm{~L} 1$ ) that was maintained at a low frequency in subsequent passages of the virus population.

\section{Whole-genome sequencing of the evolved lineages}

All evolved and the ancestral TEV-eGFP lineages were fully sequenced by Illumina technology (SRA accession: SRP075180). The consensus sequence of the ancestral TEV-eGFP population was used as a reference for mapping the evolved lineages. The deletion observed by RTPCR (Fig. 2a) in one of the D. stramonium lineages was confirmed by a low number of reads mapping inside the eGFP gene (median coverage: 111.5), compared to a higher average coverage outside this region (median coverage $P 1$ gene: 19190 , median overall genome coverage: 18460). The large deletion included the $\mathrm{N}$-terminal region of HC-Pro, as observed for other deletions that occur after gene insertions before this gene [21, 36]. For all other lineages in D. stramonium and N. benthamiana, coverage over the genome was largely uniform and similar to the ancestral virus population, indicating that there 


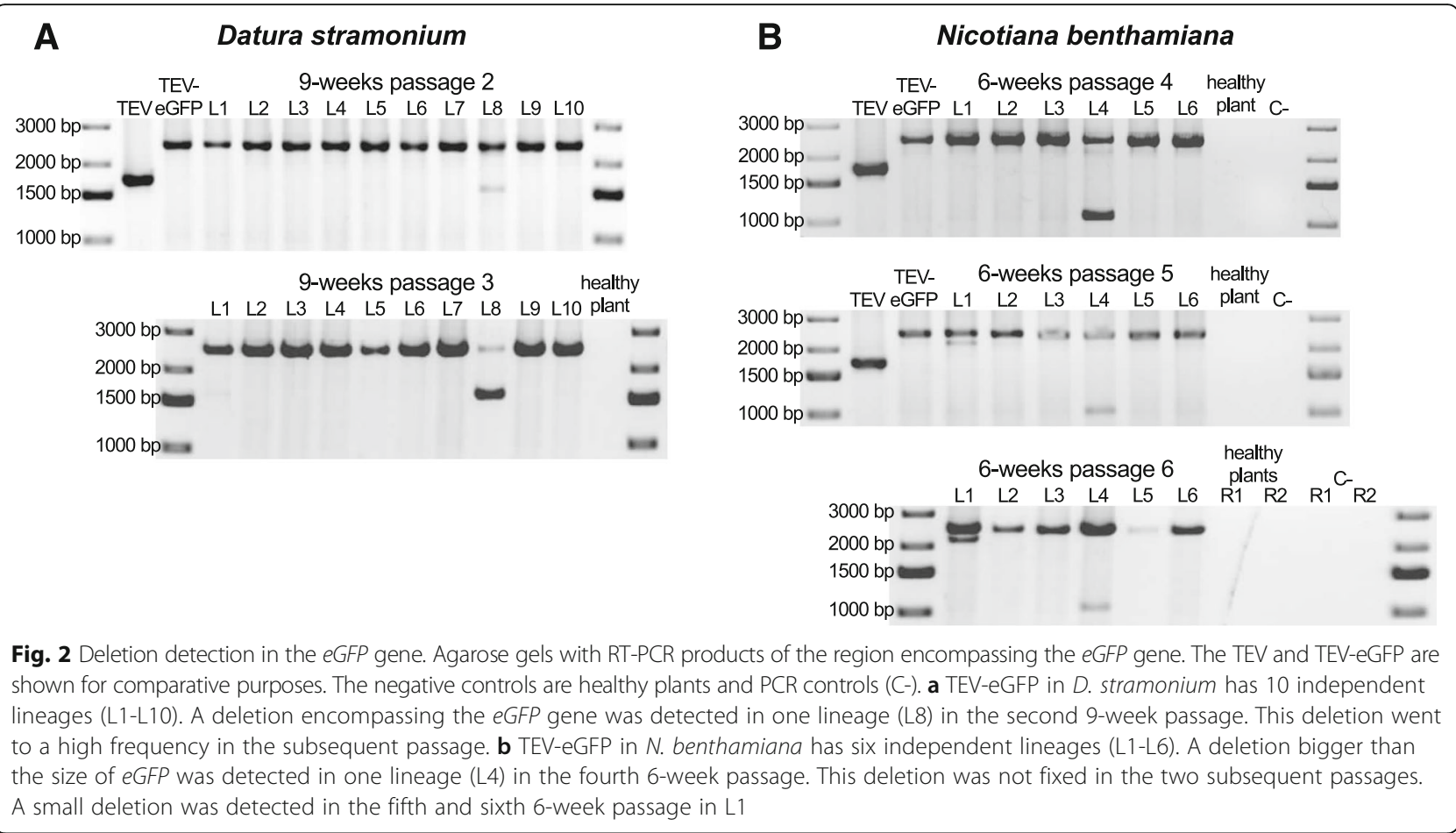

were indeed no genomic deletions present at appreciable frequencies.

Single nucleotide mutations were detected from a frequency as low as $1 \%$, comparing the evolved TEV-eGFP lineages in $N$. benthamiana and $D$. stramonium to the ancestral population (Fig. 3). This detection was also performed for evolved TEV-eGFP lineages in $N$. tabacum, that were sequenced in a previous study [21] (SRA accession: SRP075228). In the evolved $N$. benthamiana lineages 165 unique mutations were found, with a median of 34.5 (range: $27-47$ ) mutations per lineage. In the evolved $D$. stramonium lineages 239 unique mutations were found, with a median of 31.5 (range: 16-35) mutations per lineage. In the evolved $N$. tabacum lineages, 183 unique mutations were found, with a median of 21.5 (range: 17-36) mutations per lineage. Note that fixed single nucleotide mutations (frequency $>50 \%$ ) are also detected in the evolved lineages (squared symbols in Fig. 3), as the detection was done over the ancestral population. Hence, it allows us to compare the mutations that arose by evolving TEV-eGFP in the different hosts.

We detected only one mutation (U6286C; CI/Y2096H) that is shared between all three hosts. However, this mutation was present at a low frequency and not detected in all D. stramonium and N. tabacum lineages (Fig. 3 and Table 1). The $N$. benthamiana and D. stramonium lineages share more mutations (15) than either $N$. benthamiana or D. stramonium share with $N$. tabacum (4 and 9, respectively). However, most of these mutations are present in only a few lineages and at low frequency (Fig. 3 and Table 1).

The synonymous mutations U7092C, A7479C and A8253C, that are shared between D. stramonium and $N$. tabacum, are present in the highest number of lineages and reach higher frequencies among all shared mutations detected in the three hosts. These mutations were already present in the ancestral population, nevertheless, the frequencies at which these mutations are present display interesting patterns. In both $D$. stramonium and $N$. tabacum the mutations A7479C and A8253C are always detected at the same frequency within each lineage, suggesting a strong linkage between them (Additional file 1: Fig. S1). Furthermore, the U7092C mutation never appears together with the former two mutations (Additional file 1: Fig. S1), suggesting that this mutation occurs in another haplotype. Interestingly, the ancestral U7092C, A7479C and A8253C mutations were not detected in the $N$. benthamiana lineages, demonstrating the differences in host-pathogen interactions. Moreover, mutation A7567G, which was not present in the ancestral population, also appears only in the $D$. stramonium and $N$. tabacum lineages (Additional file 1: Fig. S1).

Host-specific mutations were mostly found in the evolved TEV-eGFP lineages of $N$. benthamiana (Fig. 3 and Table 2). In this host, a total number of 7 specific mutations were detected, all of them being nonsynonymous. In $D$. stramonium no host-specific mutations were detected. In $N$. tabacum only one host-specific mutation was detected in the 3'UTR (Table 2). Note that host 


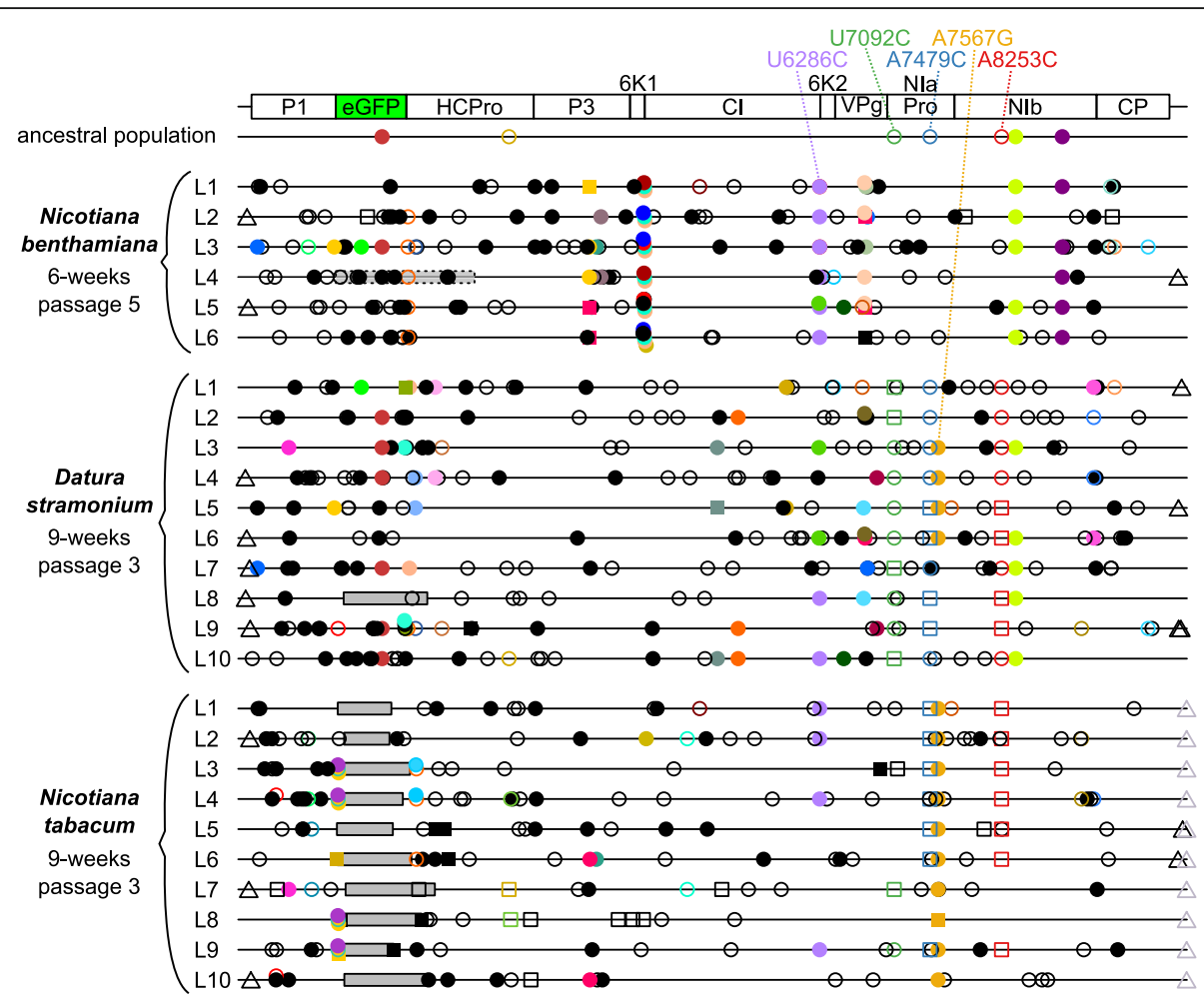

Fig. 3 Genomes of the TEV-eGFP lineages evolved the three different hosts as compared to the ancestral lineage. Mutations were detected using NGS data of the evolved lineages (L1-L10), as compared to their ancestral population. The square symbols represent mutations that are fixed $(>50 \%)$ and the circle symbols represent mutations that are not fixed $(<50 \%)$. Filled symbols represent nonsynonymous substitutions and open symbols represent synonymous substitutions. The triangle symbols represent mutations that are present in either the 3'UTR or 5'UTR. Black substitutions occur only in one lineage, whereas color-coded substitutions are repeated in two or more lineages. Note that the mutations are present at different frequencies as reported by VarScan 2. Grey boxes with continuous black lines indicate genomic deletions in the majority variant of the virus population. The grey transparent box with dotted black lines in $L 4$ of $N$. benthamiana indicates a genomic deletion in a minority variant. The latter box was drawn to indicate the size of the deletion, assuming that the deletion starts at the first position of eGFP. The mutations discussed in the manuscript are highlighted on the top. For more information on the frequency of the mutations please see Additional file 2: Tables S1-S3

specific mutations were defined as mutations detected in at least half of the evolved lineages. For more information on the mutations found in the three hosts please see Additional file 2: Tables S1-S3.

\section{Viral accumulation and within-host competitive fitness}

We measured virus accumulation $10 \mathrm{dpi}$, by RT-qPCR for a region within the coat protein gene $(C P)$. In both host species, we found no statistically significant differences ( $t$-test with Holm-Bonferroni correction) between TEV, TEV-eGFP and the lineages of TEV-eGFP evolved in that host (Fig. 4).

We then measured within-host competitive fitness by means of head-to-head competition experiments with TEV-mCherry, a virus with a different marker but similar fitness to TEV-eGFP [23]. When serially passaging a virus in a single host plant, the outcome of this assay probably better reflects viral fitness than accumulation, because the relative and not absolute numbers of virus variants present will determine which variants dominate
[21]. Here we observed interesting differences between TEV and TEV-eGFP in the two different hosts. Whereas the TEV-eGFP had lower fitness than the wild-type virus in D. stramonium (Fig. 5b, compare TEV and ancestral TEV-eGFP; $t$-test: $\left.t_{4}=13.438, P<0.001\right)$, there was no difference in $N$. benthamiana (Fig. 5b, compare TEV and ancestral TEV-eGFP; $t$-test: $t_{4}=-1.389, P=0.237$ ). Our results therefore suggest that although there is a fitness cost associated with the eGFP gene in N. tabacum [21] and D. stramonium, there is none in $N$. benthamiana. Interestingly, $N$. tabacum and $N$. benthamiana are more closely related to each other than either species is to $D$. stramonium, and yet the host species has a strong effect on the costs of a heterologous gene. This observation clearly clashes with our expectation that $e G F P$ would have a fitness cost.

For only $1 / 10$ of the evolved lineages in $D$. stramonium, we observed a significant increase in competitive fitness compared to the ancestral TEV-eGFP (Fig. 5b, L8; $t$-test with Holm-Bonferroni correction: $t_{4}=-6.890, P=0.002$ ). 
Table 1 TEV-eGFP mutations shared in the different hosts

\begin{tabular}{|c|c|c|c|c|c|c|c|c|}
\hline \multirow[b]{2}{*}{ Nucleotide change } & \multirow[b]{2}{*}{ Amino acid change } & \multirow[b]{2}{*}{ Gene } & \multicolumn{2}{|c|}{ N. benthamiana } & \multicolumn{2}{|l|}{ D. stramonium } & \multicolumn{2}{|l|}{ N. tabacum } \\
\hline & & & $\begin{array}{l}\text { Number of } \\
\text { lineages }\end{array}$ & Frequency range & Number of lineages & Frequency range & $\begin{array}{l}\text { Number of } \\
\text { lineages }\end{array}$ & $\begin{array}{l}\text { Frequency } \\
\text { range }\end{array}$ \\
\hline U6286C & Y2096H & $\mathrm{Cl}$ & $6 / 6$ & $0.013-0.131$ & $2 / 10$ & $0.012-0.031$ & $4 / 10$ & $0.019-0.140$ \\
\hline A208G & M70V & P1 & $1 / 6$ & 0.012 & $1 / 10$ & 0.010 & - & - \\
\hline C1039U & $\mathrm{H} 347 \mathrm{Y}$ & P1 & $1 / 6$ & 0.013 & $1 / 10$ & 0.035 & - & - \\
\hline G1332A & M444I & eGFP & $1 / 6$ & 0.176 & $1 / 10$ & 0.139 & - & - \\
\hline U1556G ${ }^{a}$ & V519G & eGFP & $1 / 6$ & 0.011 & $6 / 10$ & $0.010-0.016$ & - & - \\
\hline U1836G & synonymous & HC-Pro & $5 / 6$ & $0.093-0.108$ & $1 / 10$ & 0.089 & - & - \\
\hline A1917G & synonymous & HC-Pro & $1 / 6$ & 0.015 & $1 / 10$ & 0.017 & - & - \\
\hline A6278G & E2093G & $\mathrm{Cl}$ & $1 / 6$ & 0.012 & $2 / 10$ & $0.014-0.031$ & - & - \\
\hline C6547U & H2183Y & $V P g$ & $1 / 6$ & 0.013 & $1 / 10$ & 0.014 & - & - \\
\hline U6747C & synonymous & $V P g$ & $1 / 6$ & 0.012 & $1 / 10$ & 0.110 & - & - \\
\hline A6776G & D2259G & $V P g$ & $2 / 6$ & $0.533-0.746$ & $1 / 10$ & 0.023 & - & - \\
\hline G6803A & S2268N & $V P g$ & $1 / 6$ & 0.014 & $1 / 10$ & 0.024 & - & - \\
\hline A6438G & synonymous & $6 K 2$ & $1 / 6$ & 0.012 & $1 / 10$ & 0.024 & - & - \\
\hline$C 8405 G^{a}$ & T2802R & Nlb & $5 / 6$ & $0.010-0.018$ & $5 / 10$ & $0.010-0.016$ & - & - \\
\hline U9474C & synonymous & $C P$ & $1 / 6$ & 0.013 & $1 / 10$ & 0.061 & - & - \\
\hline C9837U & synonymous & $C P$ & $1 / 6$ & 0.070 & $1 / 10$ & 0.010 & - & - \\
\hline U3803C & 11268T & P3 & $2 / 6$ & $0.656-0.881$ & - & - & $2 / 10$ & $0.010-0.019$ \\
\hline U3872C & V2191A & P3 & $1 / 6$ & 0.064 & - & - & $1 / 10$ & 0.016 \\
\hline G4411A & V1471I & $\mathrm{Cl}$ & $1 / 6$ & 0.030 & - & - & $1 / 10$ & 0.016 \\
\hline C4989U & synonymous & $\mathrm{Cl}$ & $1 / 6$ & 0.018 & - & - & $1 / 10$ & 0.066 \\
\hline C548U & T183I & P1 & - & - & $1 / 10$ & 0.011 & $1 / 10$ & 0.024 \\
\hline$G 2928 A^{a}$ & synonymous & HC-Pro & - & - & $1 / 10$ & 0.017 & $1 / 10$ & 0.999 \\
\hline U7092 $C^{a}$ & synonymous & Nla-Pro & - & - & $10 / 10$ & $0.091-0.755$ & $2 / 10$ & $0.176-0.999$ \\
\hline A7479C ${ }^{a}$ & synonymous & Nla-Pro & - & - & $10 / 10$ & $0.132-0.790$ & $7 / 10$ & $0.960-0.999$ \\
\hline A7567G & K2523E & Nla-Pro & - & - & $4 / 10$ & $0.012-0.053$ & $10 / 10$ & $0.015-0.871$ \\
\hline G7710A & synonymous & Nla-Pro & - & - & $1 / 10$ & 0.014 & $1 / 10$ & 0.024 \\
\hline $\mathrm{A} 8253 \mathrm{C}^{\mathrm{a}}$ & synonymous & $\mathrm{N} / \mathrm{b}$ & - & - & $10 / 10$ & $0.136-0.801$ & $7 / 10$ & $0.805-0.998$ \\
\hline G9117A & synonymous & $\mathrm{N} / \mathrm{b}$ & - & - & $1 / 10$ & 0.321 & $2 / 10$ & $0.022-0.025$ \\
\hline U9249C & synonymous & $\mathrm{Nlb}$ & - & - & $2 / 10$ & $0.011-0.231$ & $1 / 10$ & 0.040 \\
\hline
\end{tabular}

${ }^{\mathrm{a} A l s o}$ detected in the ancestral population

This lineage is the only one to have a deletion in the eGFP insert. In $N$. benthamiana, 1/6 lineages had a significant increase in within-host fitness (Fig. 5a, L4; $t$-test with Holm-Bonferroni correction: $\left.t_{4}=-5.349, P=0.006\right)$. However, this increase in fitness probably is not associated with the large genomic deletion for three reasons: (i) the wildtype TEV without the eGFP gene has a similar fitness compared to the ancestral TEV-eGFP, suggesting deletions in $e$ GFP would not be beneficial, (ii) the RT-PCR results show that this variant occurs at a low frequency in the population, and therefore is unlikely to affect strongly the results of the competition assay, and (iii) this deletion variant remains at low frequency during the next round of passaging (Fig. 2b), suggesting that while frequency-dependent selection might occur, its fitness is not higher than the coevolving full-length TEV-eGFP. Moreover, another lineage of $N$. benthamiana where we did not detect any deletions, also appeared to have increased in fitness (Fig. 5a, L6; $t$-test: $t_{4}=-4.0792, P=0.015$ ), however, after the Holm-Bonferroni correction not significantly. Interestingly, the lineage that did increase its fitness significantly (L4) is the only lineage that contains mutations in the $6 \mathrm{~K} 2$ protein in this host (Additional file 2: Table S1). Therefore, we speculate that single-nucleotide variation is one of the main driving forces for an increase in TEV-eGFP fitness in N. benthamiana.

These fitness measurements show that most lineages failed to adapt to the new host species. However, in the 
Table 2 Host specific mutations in the evolved TEV-eGFP lineages

\begin{tabular}{|c|c|c|c|c|c|}
\hline & Nucleotide change & Amino acid change & Gene & Number of lineages & Frequency range \\
\hline \multirow[t]{7}{*}{ N. benthamiana } & G3797A & G1266E & P3 & $3 / 6$ & $0.291-0.664$ \\
\hline & G4380U & E1460D & $6 K 1$ & $3 / 6$ & $0.012-0.093$ \\
\hline & U4387C & Y1463H & $6 K 1$ & $4 / 6$ & $0.011-0.016$ \\
\hline & C4391U & T1464M & $6 K 1$ & $6 / 6$ & $0.041-0.138$ \\
\hline & G4397A & S1466N & $\mathrm{Cl}$ & $6 / 6$ & $0.012-0.019$ \\
\hline & A6771U & L2257F & $V P g$ & $4 / 6$ & $0.027-0.201$ \\
\hline & G8909U ${ }^{a}$ & W2970L & NIb & $5 / 6$ & $0.026-0.042$ \\
\hline D. stramonium & - & - & - & - & - \\
\hline N. tabacum & G10253A & & 3'UTR & $10 / 10$ & $0.025-0.040$ \\
\hline
\end{tabular}

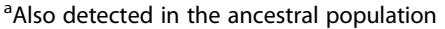

two cases that there were significant fitness increases, the underlying genetic changes were consistent with the expected route of adaptation, when our expectations were modified based on the unexpected results for the competitive fitness of the ancestral TEV-eGFP virus in the two alternative host species. In $D$. stramonium, where carrying eGFP imposes a high fitness cost, this sequence was deleted. In $N$. benthamiana, where carrying eGFP apparently has not fitness cost, host-specific single-nucleotide variation was observed.

\section{Discussion}

We set out to explore the hypothesis that differences in virulence for different hosts could have an effect on the rate of virus adaptation in each host [13]. Although we find this hypothesis simple and provocative, the observed patterns in our experiments suggest that even in a controlled laboratory environment, the biological reality will often be complex and hard to predict. We used a virus expressing an eGFP fluorescent marker in the hope that the loss of this marker could serve as a real-time

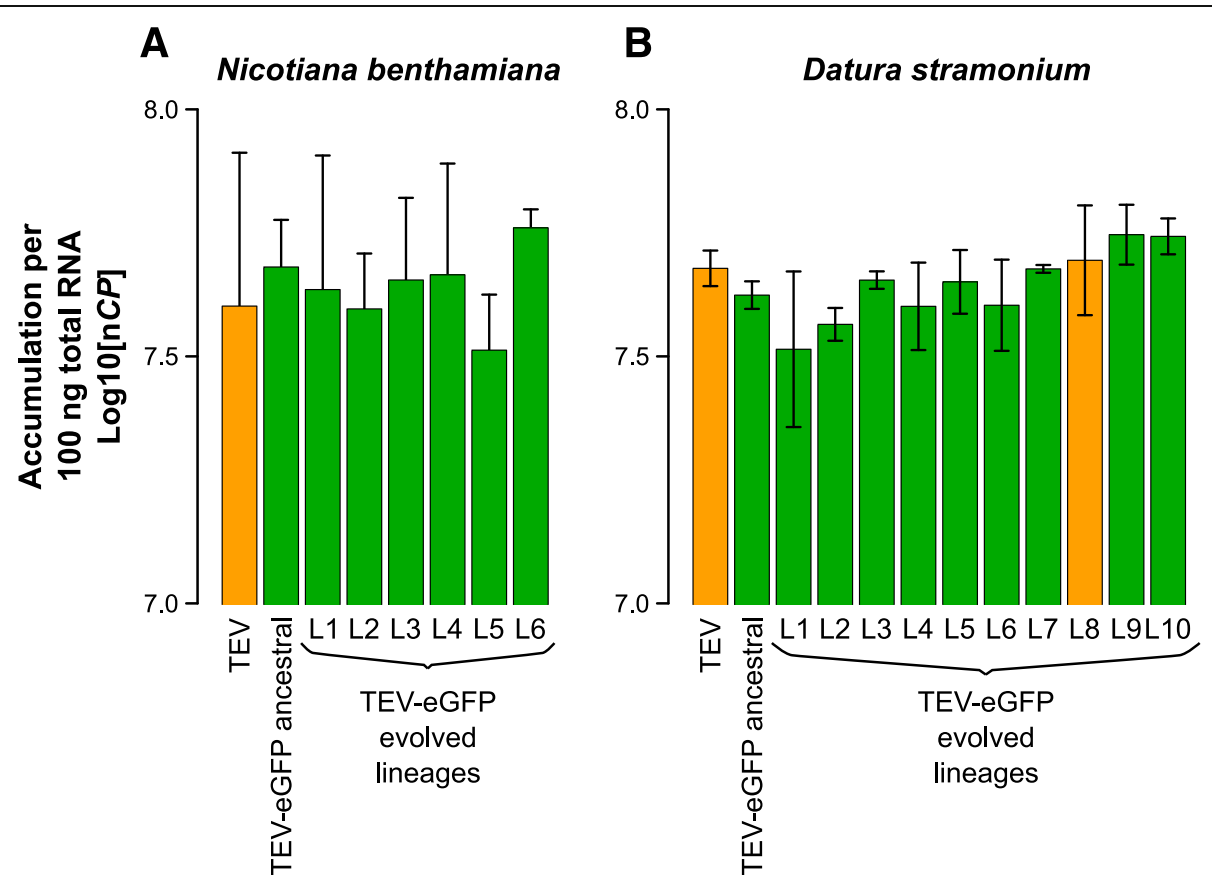

Fig. 4 Virus accumulation of the evolved and ancestral lineages. Virus accumulation, as determined by accumulation experiments and RT-qPCR at $10 \mathrm{dpi}$, of TEV, the ancestral TEV-eGFP, and the evolved TEV-eGFP lineages in N. benthamiana (a) and D. stramonium (b). TEV and the evolved lineage with a deletion in the eGFP gene (B; L8) are indicated with the orange bars. The ancestral TEV-eGFP and the evolved lineages with an intact eGFP gene are indicated with the green bars. No significant differences between TEV and TEV-eGFP ancestral were found, nor between these and the evolved lineages ( $t$-test with Holm-Bonferroni correction for multiple tests). Error bars represent SD of the plant replicates 
A

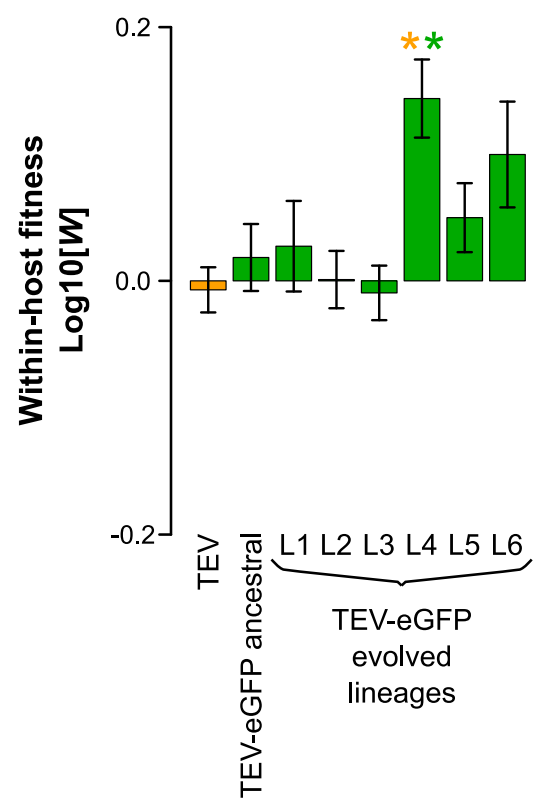

B

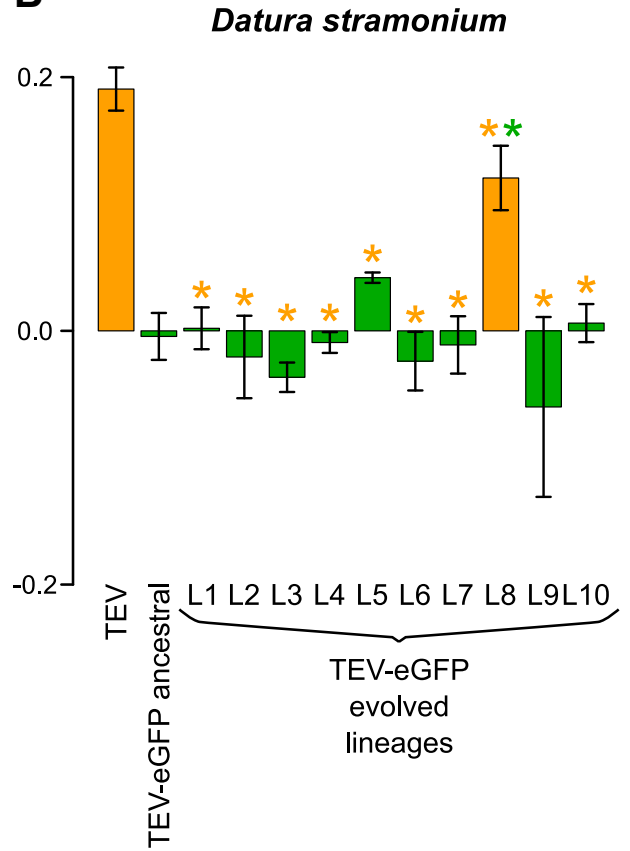

Fig. 5 Within-host competitive fitness of the evolved and ancestral lineages. Fitness $(W$ ) determined by competition experiments and RT-qPCR of the different viral genotypes with respect to a common competitor; TEV-mCherry. W was determined at $10 \mathrm{dpi}$, of TEV, the ancestral TEV-eGFP, and the evolved TEV-eGFP lineages in N. benthamiana (a) and D. stramonium (b). TEV and the evolved lineage with a deletion in the eGFP gene $(B ; L 8)$ are indicated with the orange bars. The ancestral TEV-eGFP and the evolved lineages with an intact eGFP gene are indicated with the green bars. The orange asterisks indicate statistical significant differences of the evolved lineages as compared to TEV (t-test with Holm-Bonferroni correction for multiple tests). The green asterisks indicate statistical significant differences of the evolved lineages as compared to the ancestral TEV-eGFP ( $t$-test with Holm-Bonferroni correction for multiple tests). Not indicated in the figure is that TEV and TEV-eGFP ancestral tested significantly different in D. stramonium ( $B$; $t$-test: $t_{4}=13.438, P<0.001$ ) while not in $N$. benthamiana $\left(A ; t\right.$-test: $t_{4}=-1.389, P=0.237$ ). Error bars represent $S D$ of the plant replicates

indicator of adaptation. Even though this method served us in the past in the natural host N. tabacum [21], there were complications with this method in the two alternative hosts used in this study, and a loss of fluorescence was only observed in a single $D$. stramonium lineage. RT-PCR and Illumina sequencing confirmed the loss of the eGFP marker in this case, and its integrity in all other lineages. The data of our competitive fitness assay demonstrate why the marker sequence was probably rather stable in N. benthamiana; eGFP does not appear to have a cost in this host species, in sharp contrast to the strong fitness cost observed in D. stramonium as well as previously observed in the more closely related host $N$. tabacum [21]. We expect that the marker will eventually be lost, but only due to genetic drift and therefore at a slow rate. The observed lack of fitness cost for eGFP in $N$. benthamiana was an unexpected result, and further undermined our initial idea of using the loss of this gene as a real-time indicator of virus adaptation.

What mechanisms might underlie the difference in the fitness costs of eGFP marker in these two host plants? Does the difference in passage length used influences the rate of marker loss in $N$. benthamiana and $D$. stramonium? In a previous study, we showed that the loss of the eGFP marker occurred more rapidly as the duration of each passage was increased [21]. During long passages transmission bottlenecks are more spaced in time, and much larger census population sizes are reached. Moreover, longer passages also give a much greater opportunity for virus movement into the newly developing host tissues. This does not apply to $N$. benthamiana; due to the high virulence of TEV, these plants did not grow much and eventually died. During passaging of TEV-eGFP, leaves were therefore harvested after 6 weeks, just before plants were completely dead. We think that the marked differences in virus-host interactions will be the main determinants of virus evolution and marker stability, although we cannot rule out categorically that the differences in passage length also might have played a role. As for N. tabacum [21], here we again observed that the eGFP marker does not affect virus accumulation, whereas it does lower competitive fitness in D. stramonium. These observations suggest that the effects of the eGFP marker on virus movement are the main reason for selection against the marker, as for TEV cellular co-infections are rare and therefore fast within-host spread is crucial to invade more space and hereby having a 
higher competitive fitness [37]. However, marker loss in $D$. stramonium appears to occur more slowly compared to $N$. tabacum [21], indicating poor virus adaptation to this alternative host. Given the high virulence of TEV for $N$. benthamiana, including strong stunting, there will be limited virus movement during infection and thus significantly lower population census sizes. Hence, we speculate that in $N$. benthamiana the limited scope for virus movement and accumulation - due to the virus' virulence itself - might mitigate the cost of the eGFP marker. Alternatively, cell-tocell and systemic virus movement in $N$. benthamiana might be so slow that the addition of the eGFP marker matters little. A slow systemic virus movement may also explain why in the second round of infection four lineages failed to re-infect $N$. benthamiana, as initial virus accumulation appeared to be very low until possible virus adaptation by means of point mutations occurred.

These results are at odds with our expectations, but they nevertheless have some interesting implications. First, host species changes can apparently ameliorate the costs of exogenous genes. Although strong virus genotype-by-host species interactions have been previously shown for TEV [17], we did not anticipate that a such a simple difference (the presence of $e G F P$ ) could also be subjected to such an interaction. These results suggest that when considering the evolution of genome architecture, host species might play a very important role, by allowing evolutionary intermediates to be competitive. For example, for TEV we have shown that the evolution of an alternative gene order through duplication of the NIb replicase gene is highly unlikely, as this intermediate step leads to significant decreases in fitness, making the trajectory to alternative gene orders inaccessible [36]. We have also shown that potentially beneficial gene duplications in the TEV genome are selected against, as these also lead to a significant reduction in viral fitness [38]. If gene duplication has a similar interaction with host species as the eGFP insert has, then an alternative host species could act as a stepping-stone and hereby increase the accessibility of the evolutionary trajectories to alternative genome architectures. Similar effects of environmental change have been noted in other studies [39]. The generality of these results has not been addressed yet using other viruses with altered genome architecture, but the possibilities are tantalizing. Second, our results could also have implications for assessing the biosafety risks of the genetically modified organisms. Our results suggest that extrapolating fitness results from a permissive host to alternative hosts can be problematic, even when the scope for unexpected interactions appears to be limited, as would be the case for the addition of eGFP expression. In other model systems, unexpected interactions between heterologous genes and host species have also been reported [40].
Upon passaging in each alternative host species, only a single lineage evolved higher fitness. The low rate of adaptation observed was consistent with a previous report [18], although we used passages of a longer duration here and had therefore expected more rapid adaptation [21]. Given the low rate at which lineages adapted in this experiment, however, although the rate of adaptation appears to be similar in both hosts, we do not consider that our results allow for a good test of our hypothesis. Nevertheless, our results do stress that differences in host biology can have a much stronger effect on evolutionary dynamics than differences in virus-induced virulence between host species. An alternative way to tackling the question of the effects of virulence on adaptation might be to use a biotechnological approach; hosts which have different levels of virulence can be engineered, to ensure the main difference between host treatment is microparasite-induced virulence. For example, plant hosts could be engineered to express antiviral siRNAs at low levels. Such an approach would allow for a more controlled test of the hypothesis suggested here, whilst probably not being representative for natural host populations. On the other hand, such experiments could perhaps help shed light on the effects of virulence on adaptation in agroecosystems or vaccinated populations.

\section{Conclusions}

A host species jump can be a game changer for evolutionary dynamics. An exogenous sequence - eGFP - which is unstable in its typical host, has shown to be more stable in two alternative host species for which TEV has both lower and higher virulence than in the typical host. In addition, eGFP does not appear to have any fitness effects in the host for which TEV has high virulence. These observations clashed with the hypothesis that high virulence slows down the rate of adaptation. Moreover, when considering the evolution of genome architecture, host species jumps might play a very important role, by allowing evolutionary intermediates to be competitive.

\section{Additional files}

Additional file 1: Figure S1. Frequency of mutations found in both $D$. stramonium and $N$. tabacum. Mutations detected in both D. stramonium and $N$. tabacum that were present in all the lineages of either one of these hosts. The frequency of these mutations in either the ancestral population (anc) or the different lineages (L1-L10) is given by the color-coded points. (PDF $44 \mathrm{~kb}$ )

Additional file 2: Table S1. Mutations detected in the $N$. bentiamiana lineages as compared to the ancestral lineage. Table S2. Mutations detected in the $D$. stramonium lineages as compared to the ancestral lineage. Table S3. Mutations detected in the N. tabacum lineages as compared to the ancestral lineage. (XLS $149 \mathrm{~kb}$ )

Acknowledgements

We thank Francisca de la Iglesia and Paula Agudo for excellent technical assistance. 


\section{Funding}

This work was supported by the John Templeton Foundation [grant number 22371 to S.F.E]; the European Commission $7^{\text {th }}$ Framework Program EvoEvo Project [grant number ICT-610427 to S.F.E.]; and the Spanish Ministerio de Economía y Competitividad (MINECO) [grant numbers BFU2012-30805 and BFU2015-65037-P to S.F.E]. The opinions expressed in this publication are those of the authors and do not necessarily reflect the views of the John Templeton Foundation. The funders had no role in study design, data collection and analysis, decision to publish, or preparation of the manuscript.

\section{Availability of data and material}

The raw read data from Illumina sequencing is available at SRA with accession: SRP075180. The fitness data has been deposited on LabArchives with doi: 10.6070/H4N877TD.

\section{Authors' contributions}

AW, MPZ and SFE designed the study. AW and MPZ performed the experiments. AW, MPZ and SFE analyzed the data and wrote the manuscript. All authors read and approved the final manuscript.

\section{Competing interests}

The authors declare that they have no competing interests.

\section{Consent for publication}

Not applicable.

\section{Ethics approval and consent to participate}

Not applicable.

\section{Author details}

'Instituto de Biología Molecular y Celular de Plantas (IBMCP), Consejo Superior de Investigaciones Científicas-Universidad Politécnica de Valencia, Campus UPV CPI 8E, Ingeniero Fausto Elio s/n, 46022 València, Spain. 2Present address: MIVEGEC (UMR CNRS 5290, IRD 224, UM), National Center for Scientific Research (CNRS), 911 Avenue Agropolis, BP 64501, 34394 Cedex 5 Montpellier, France. ${ }^{3}$ Present address: Institute of Theoretical Physics, University of Cologne, Zülpicher Straße 77, 50937 Cologne, Germany. ${ }^{4}$ The Santa Fe Institute, 1399 Hyde Park Road, Santa Fe, NM 87501, USA.

\section{Received: 21 June 2016 Accepted: 11 January 2017}

Published online: 19 January 2017

\section{References}

1. Ewald PW. Host-parasite relations, vectors, and the evolution of disease severity. Annu Rev Ecol Syst. 1983;14:465-85

2. Alizon S, Hurford A, Mideo N, Van Baalen M. Virulence evolution and the trade-off hypothesis: history, current state of affairs and the future. J Evol Biol. 2009:22:245-59.

3. Fraser C, Hollingsworth TD, Chapman R, de Wolf F, Hanage WP. Variation in HIV-1 set-point viral load: epidemiological analysis and an evolutionary hypothesis. Proc Natl Acad Sci U S A. 2007;104:17441-6.

4. De Roode JC, Yates AJ, Altizer S. Virulence-transmission trade-offs and population divergence in virulence in a naturally occurring butterfly parasite. Proc Natl Acad Sci U S A. 2008;105:7489-94.

5. Mackinnon MJ, Gandon S, Read AF. Virulence evolution in response to vaccination: the case of malaria. Vaccine. 2008;26 Suppl 3:C42-52.

6. Ebert D, Weisser WW. Optimal killing for obligate killers: the evolution of life histories and virulence of semelparous parasites. Proc R Soc B. 1997;264:985-91.

7. Pagán I, Montes N, Milgroom MG, García-Arenal F. Vertical transmission selects for reduced virulence in a plant virus and for increased resistance in the host. PLoS Pathog. 2014;10:e1004293.

8. May RM, Nowak MA. Coinfection and the evolution of parasite virulence. Proc R Soc B. 1995;261:209-15.

9. De Roode JC, Pansini R, Cheesman SJ, Helinski MEH, Huijben S, Wargo AR, Bell AS, Chan BHK, Walliker D, Read AF. Virulence and competitive ability in genetically diverse malaria infections. Proc Natl Acad Sci U S A. 2005;102:7624-8.

10. Brown SP, Cornforth DM, Mideo N. Evolution of virulence in opportunistic pathogens: generalism, plasticity, and control. Trends Microbiol. 2012; 20:336-42.

11. Boots M, Sasaki A. The evolutionary dynamics of local infection and globa reproduction in host-parasite interactions. Ecol Lett. 2000;3:181-5.
12. Bull JJ, Lauring AS. Theory and empiricism in virulence evolution. PLoS Pathog. 2014;10:e1004387.

13. Bull JJ, Ebert D. Invasion thresholds and the evolution of nonequilibrium virulence. Evol Appl. 2008;1:172-82.

14. Lenski RE, May RM. The evolution of virulence in parasites and pathogens: reconciliation between two competing hypotheses. J Theor Biol. 1994; 169:253-65.

15. Leroy EM, Kumulungui $B$, Pourrut $X$, Rouquet $P$, Hassanin $A$, Yaba $P$, Délicat A, Paweska JT, Gonzalez J-P, Swanepoel R. Fruit bats as reservoirs of Ebola virus. Nature. 2005;438:575-6.

16. Longdon B, Hadfield JD, Day JP, Smith SCL, McGonigle JE, Cogni R, Cao C, Jiggins FM. The causes and consequences of changes in virulence following pathogen host shifts. PLoS Pathog. 2015;11:e1004728.

17. Lalić J, Cuevas JM, Elena SF. Effect of host species on the distribution of mutational fitness effects for an RNA virus. PLoS Genet. 2011:7:e1002378.

18. Bedhomme S, Lafforgue G, Elena SF. Multihost experimental evolution of a plant RNA virus reveals local adaptation and host-specific mutations. Mol Biol Evol. 2012;29:1481-92.

19. Velasquez N, Hossain MJ, Murphy JF. Differential disease symptoms and fulllength genome sequence analysis for three strains of Tobacco etch virus. Virus Genes. 2015:50:442-9.

20. Revers F, García JA. Molecular biology of potyviruses. Adv Virus Res. 2015:92:101-99.

21. Zwart MP, Willemsen A, Daròs JA, Elena SF. Experimental evolution of pseudogenization and gene loss in a plant RNA virus. Mol Biol Evol. 2014:31:121-34.

22. Carrington JC, Haldeman R, Dolja W, Restrepo-Hartwig MA. Internal cleavage and trans-proteolytic activities of the VPg-proteinase (Nla) of tobacco etch potyvirus in vivo. J Virol. 1993;67:6995-7000.

23. Zwart MP, Daròs JA, Elena SF. One is enough: in vivo effective population size is dose-dependent for a plant RNA virus. PLoS Pathog. 2011;7:e1002122.

24. Carrasco P, Daròs JA, Agudelo-Romero P, Elena SF. A real-time RT-PCR assay for quantifying the fitness of Tobacco etch virus in competition experiments. Virol Methods. 2007:139:181-8.

25. FASTX-Toolkit. Hannon Lab. http://hannonlab.cshl.edu/fastx_toolkit/index. html. Accessed 4 Oct 2016.

26. Schmieder R, Edwards R. Quality control and preprocessing of metagenomic datasets. Bioinformatics. 2011:27:863-4.

27. Langmead B, Salzberg SL. Fast gapped-read alignment with Bowtie 2. Nat Methods. 2012:9:357-9.

28. Li H, Handsaker B, Wysoker A, Fennell T, Ruan J, Homer N, Marth G, Abecasis $\mathrm{G}$, Durbin R. The sequence alignment/map format and SAMtools. Bioinformatics. 2009;25:2078-9.

29. Koboldt DC, Zhang Q, Larson DE, Shen D, McLellan MD, Lin L, Miller CA Mardis ER, Ding L, Wilson RK. VarScan 2: Somatic mutation and copy number alteration discovery in cancer by exome sequencing. Genome Res. 2012:22:568-76.

30. R Core Team. R: A language and environment for statistical computing. Vienna, Austria: R Foundation for Statistical Computing; 2014. https://www.rproject.org/. Accessed 4 Oct 2016.

31. Dolja W, Herndon KL, Pirone TP, Carrington JC. Spontaneous mutagenesis of a plant potyvirus genome after insertion of a foreign gene. J Virol. 1993:67:5968-75

32. Majer E, Daròs JA, Zwart M. Stability and fitness impact of the visually discernible Roseal marker in the tobacco etch virus genome. Viruses. 2013;5:2153-68.

33. Plisson C, Drucker M, Blanc S, German-Retana S, Le Gall O, Thomas D, Bron P. Structural characterization of HC-Pro, a plant virus multifunctional protein. J Biol Chem. 2003;278:23753-61.

34. Shiboleth YM, Haronsky E, Leibman D, Arazi T, Wassenegger M, Whitham SA, Gaba V, Gal-On A. The conserved FRNK box in HC-Pro, a plant viral suppressor of gene silencing, is required for small RNA binding and mediates symptom development. J Virol. 2007;81:13135-48.

35. Sardanyés J, Elena SF. Error threshold in RNA quasispecies models with complementation. J Theor Biol. 2010;265:278-86

36. Willemsen A, Zwart MP, Tromas N, Majer E, Daròs JA, Elena SF. Multiple barriers to the evolution of alternative gene orders in a positive-strand RNA virus. Genetics. 2016:202:1503-21.

37. Tromas N, Zwart MP, Lafforgue G, Elena SF. Within-host spatiotemporal dynamics of plant virus infection at the cellular level. PLoS Genet. 2014;10:e1004186. 
38. Willemsen A, Zwart MP, Higueras P, Sardanyés J, Elena SF. Predicting the stability of homologous gene duplications in a plant RNA virus. Genome Biol Evol. 2016;8:3065-82.

39. De Vos MGJ, Dawid A, Sunderlikova V, Tans SJ. Breaking evolutionary constraint with a tradeoff ratchet. Proc Natl Acad Sci U S A. 2015;112:14906-11.

40. Hernández-Crespo P, Sait SM, Hails RS, Cory JS. Behavior of a recombinant baculovirus in lepidopteran hosts with different susceptibilities. Appl Environ Microbiol. 2001;67:1140-6.

Submit your next manuscript to BioMed Central and we will help you at every step:

- We accept pre-submission inquiries

- Our selector tool helps you to find the most relevant journal

- We provide round the clock customer support

- Convenient online submission

- Thorough peer review

- Inclusion in PubMed and all major indexing services

- Maximum visibility for your research

Submit your manuscript at www.biomedcentral.com/submit 